Encontros Bibli: revista eletrônica de biblioteconomia e ciência da informação, $v .17, n$. esp. 2 - III SBCC, p.36-54, 2012. ISSN 1518-2924. DOI:10.5007/15182924.2012v17nesp $2 p 36$

\title{
SERVIÇOS DE DIFUSÃO DE POLÍTICAS PARA O ACESSO ABERTO: SHERPA/JULIET, MELIBEA E ROARMAP
}

\author{
Michelli $\operatorname{Costa}^{1}$ \\ Bianca Amaro ${ }^{2}$ \\ Tainá $\mathrm{Assis}^{3}$
}

\begin{abstract}
Resumo: Aborda os serviços de difusão de políticas para a promoção do acesso aberto à informação científica. A criação dessas políticas é fundamental para que as instituições garantam a aplicação de estratégias que objetivam tornar acessíveis publicações científicas. As instituições de pesquisa são as grandes produtoras de informação. A ampliação dos processos de socialização do conhecimento nesses ambientes colabora para a otimização da comunicação científica. Os serviços de difusão das políticas para têm como objetivo tornar conhecidas as políticas e suas iniciativas. Neste sentido, a análise desses serviços se mostra importante para ampliar o conhecimento a esse respeito, identificando seus benefícios e deficiências de forma que possam ser aprimorados e possam também fundamentar a criação de novos serviços. Foi realizado um estudo comparativo entre os serviços Sherpa/Juliet, Melibea e ROARMAP que gerou um panorama atual do que é oferecido por cada um deles, destacando as suas similaridades e especificidades. Observou-se também, que os referidos serviços cumprem com seus objetivos e oferecem subsídios para criação de novas políticas nesse contexto.
\end{abstract}

Palavras-chave: Acesso livre. Acesso livre - Região Nordeste do Brasil.

\section{DISSEMINATION SERVICES OF OPEN ACCESS POLICIES: SHERPA/JULIET, MELIBEA} AND ROARMAP

\begin{abstract}
This article discusses the diffusion of policies for promoting open access to scientific information. The creation of these policies is essential for institutions to ensure the implementation of strategies that aim to make scientific publications available. Research institutions are the major producers of information. The expansion of the processes of socialization of knowledge in these environments contributes to the optimization of scientific communication. The diffusion services of the policies for this purpose are intended to make their policies and initiatives known. Therefore, its analysis shows the importance to increase knowledge about these services by identifying its benefits and shortcomings, so that they can be improved and substantiate the creation of new services. We conducted a comparative study of the Sherpa/Juliet, Melibea ROARMAP services and generated a current overview of what is offered by each of them, highlighting their similarities and specificities. It was also observed, that those services meet their goals and provide subsidies for the creation of new policies in this context.
\end{abstract}

Keywords: Open access. Open access policies. Scientific communication.

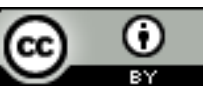

Esta obra está licenciada sob uma Licença Creative Commons

\footnotetext{
${ }^{1}$ Instituto Brasileiro de Informação em Ciência e Tecnologia (IBICT) e Universidade de Brasília (UNB) michelli@ibict.br

${ }^{2}$ Instituto Brasileiro de Informação em Ciência e Tecnologia (IBICT) - $\underline{\text { bianca@ibict.br }}$

${ }^{3}$ Instituto Brasileiro de Informação em Ciência e Tecnologia (IBICT) - taina@ibict.br
} 


\section{INTRODUÇÃO}

A comunicação científica é o processo que acontece dentro de uma comunidade científica e/ou acadêmica para a transferência e socialização do conhecimento científico. Esse processo de comunicação, segundo Kuramoto (2006), oferece meios para a interação social de uma comunidade científica e contribui para a produção, disseminação e uso do conhecimento, o que é fundamental para o avanço da ciência. A função da comunicação científica é promover melhores resultados a questões específicas, estimular a busca por novos conhecimentos, divulgar novos resultados e tendências, e avaliar a confiabilidade de novas ideias com a possibilidade de verificação, fornecimento de feedback para as produções, registro da autoria e preservação do registro do conhecimento científico (BAPTISTA et al, 2007).

Tradicionalmente, a forma de comunicação científica mais difundida é aquela realizada por meio dos periódicos. Desde o surgimento, na década de 1990, do movimento pelo acesso aberto e pela mudança no modelo tradicional das publicações, houve crescimento, em todo o mundo, de iniciativas e serviços que possibilitam a difusão das informações de forma irrestrita, livre e, principalmente, isenta de custos financeiros. Esses novos paradigmas trouxeram benefícios inequívocos ao processo da comunicação, com destaque para o aumento da agilidade na difusão da informação e na ampliação da visibilidade dos trabalhos publicados.

As instituições que promovem pesquisas são as produtoras de grande parte da informação científica e, consequentemente, possuem grande potencial para contribuir com os processos relacionados ao acesso aberto à comunicação. Há que se ter em conta que essas instituições, além de serem produtoras de informação, também são consumidoras. Logo, quando aderem a uma estratégia para o acesso aberto, estão ao mesmo tempo fortalecendo as iniciativas em um contexto global e se beneficiando dos resultados.

Neste trabalho serão apresentados os desafios que o acesso aberto ainda encontra para ganhar espaço na comunicação científica, após quase vinte anos do surgimento. Em seguida, serão abordadas as políticas e suas características que devem ser pensadas e definidas para que esses obstáculos sejam superados. Atualmente existem serviços que reúnem, analisam e disponibilizam políticas com o objetivo de fazê-las conhecidas no âmbito acadêmico e de fomentar a criação de novas diretrizes. Para um estudo comparativo no âmbito da presente análise foram selecionados os seguintes serviços: Sherpa/Juliet, Melibea e ROARMAP. Ao 
final serão apresentadas algumas considerações e uma proposta para o desenvolvimento de um novo serviço.

\section{DESAFIOS PARA O ACESSO ABERTO NA COMUNICAÇÃO CIENTÍFICA}

A chamada crise dos periódicos, ocorrida em meados dos anos 1990, resultou do aumento excessivo nos preços das assinaturas e consequentemente da dificuldade financeira das bibliotecas universitárias e dos centros de informação e pesquisa em manterem suas coleções atualizadas. Essa crise levou ao surgimento de um novo modelo de publicação, que consiste na criação e distribuição de periódicos eletrônicos por meio da Internet. Sobre essa mudança, Lima (2009, p. 222) afirma:

Como reação, a partir dos anos 1990, vemos lentamente delinear-se e crescer uma nova forma de disponibilização do saber produzido pela Ciência, possibilitada em larga escala pela Internet. [...]. Esse novo modo de publicar na Ciência trata-se do movimento pelo livre acesso.

O acesso aberto às publicações científicas é a disponibilização da comunicação científica isenta de custos, de maneira livre e irrestrita. Para que seja atingido esse objetivo, o Movimento pelo Acesso Aberto definiu, em 2001, duas estratégias: a via dourada e a via verde. A via dourada é a promoção da criação e disponibilização de periódicos científicos eletrônicos de acesso aberto na Internet. A segunda estratégia, a via verde, é a disponibilização dos resultados de pesquisa por meio de repositórios digitais.

Um dos desafios para o sucesso de ambas as estratégias são os empecilhos colocados pelas próprias editoras comerciais, as quais são detentoras, em sua maioria, dos direitos das publicações dos pesquisadores. As editoras que possuem um sistema comercial que lucra com as publicações científicas temem a perda da exclusividade de acesso aos textos, o que acarretaria em perda de receita. Portanto, elas pró́bem que estes sejam depositados nos repositórios ou disponibilizados por meio das revistas de acesso aberto, permitindo, assim, o acesso apenas para aqueles que pagam. Essa relação é discutida por Mueller (2006, p. 34), que afirma:

Com relação ao papel das editoras comerciais no processo, tais editoras são empresas poderosas, não só financeiramente, mas também politicamente, pois na medida em que são donas dos periódicos e detentoras dos copyrights dos trabalhos que esses periódicos publicam, controlam, de fato, o sistema de comunicação científica. Além disso, as editoras mais conceituadas ainda derivam poder justamente desse prestígio que lhes é atribuído pela comunidade. O discurso das editoras, ao não permitir acesso livre, é que elas protegem o autor e a integridade do texto. 
Apesar do argumento utilizado pelas editoras científicas comerciais, tem sido crescente o número de editores que está colaborando com o modelo de acesso aberto. Essa realidade pode ser observada em regiões como a América Latina, na qual, segundo Cerda Silva e Lara (2011), 99\% das editoras científicas são de acesso aberto. Isso pode ser atribuído ao fato de que a ampla disponibilização dos resultados das pesquisas, livre de barreiras financeiras, facilita a circulação desses trabalhos no meio científico e, consequentemente, faz com que sejam mais citados. Um estudo realizado em 2011 por Laakso et al apud Dearing (2012) comprova a ampliação do acesso aberto durante o período de 1993 a 2009.

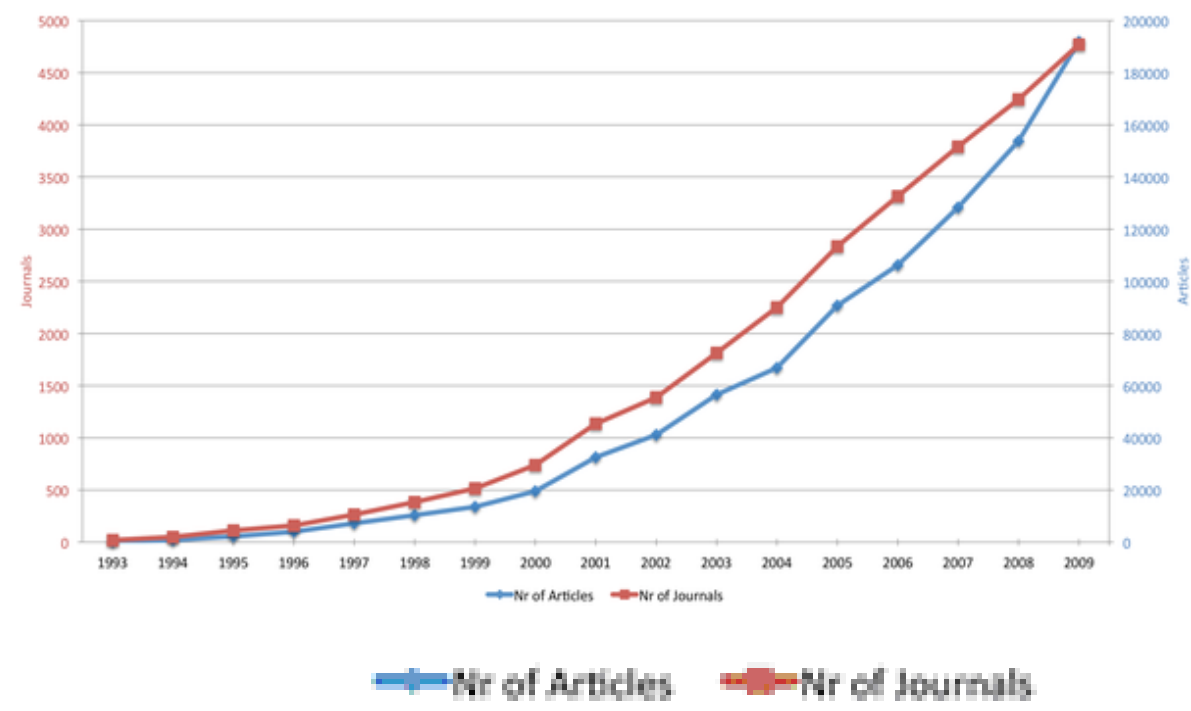

Figura 1 - Evolução do acesso aberto no período de 1993-2009.

Fonte: LAAKSO et al apud Dearing, 2012.

De acordo com Dearing (2012), durante os 17 anos utilizados para sua pesquisa, cerca de 19.500 artigos em acesso aberto estavam disponíveis em 740 periódicos no ano 2000. Em 2009, quase 192.000 artigos em acesso aberto podiam ser acessados em 4.769 periódicos científicos.

Uma das explicações para essa mudança seria a pressão dos próprios autores que estão optando por disponibilizar seus artigos em revistas de acesso aberto, visto que a visibilidade é maior. A ampliação da visibilidade dos trabalhos que estão em acesso aberto é analisada pelo trabalho de Lawrence (2001). Segundo seus resultados, os artigos online e abertos tiveram aumento de mais de 300\% em relação aos artigos publicados offline. Esta pesquisa foi realizada com mais de cem mil artigos e constatou que o número médio das citações dos artigos offline estava em torno de 2,7, enquanto que a média para as citações de artigos online chegava a 7. Sobre este resultado, o autor concluiu que para maximizar o impacto e minimizar 
a redundância e aumentar a velocidade do progresso científico, autores e editores devem ter como objetivo facilitar o acesso à pesquisa (LAWRENCE, 2001, tradução nossa) ${ }^{4}$.

Além dos próprios autores, as instituições de pesquisa e/ou financiadoras também representam parte importante no processo que impulsiona a criação de periódicos e repositórios institucionais de acordo com esta nova filosofia. Uma das formas utilizadas para esse estímulo são as políticas institucionais que requerem ou recomendam que os resultados das pesquisas que foram financiadas estejam disponíveis em acesso aberto.

Como discutido anteriormente, apesar dos avanços percebidos na atualidade, ainda são grandes os desafios para tornar mais acessíveis as publicações científicas. As mudanças nesse processo apontam para a necessidade da adoção de políticas institucionais que incentivem e fortaleçam as iniciativas com essa finalidade. Com o objetivo de contribuir para essa discussão, serão apresentados e analisados os serviços de difusão das políticas para a promoção do acesso aberto à informação científica.

\section{POLÍTICAS PARA O ACESSO ABERTO}

As políticas tratadas neste trabalho são aquelas que têm por objetivo favorecer o desenvolvimento da comunicação científica nas duas estratégias propostas pelo Movimento de Acesso Aberto. Segundo Abadal et al (2009), as políticas de uma instituição, que tem por objetivo impulsionar o acesso aberto no âmbito da comunicação científica, devem orientar, facilitar e impulsionar iniciativas nesse contexto. Na prática, segundo os autores, as políticas devem orientar e/ou facilitar para que o pesquisador arquive seus trabalhos em repositórios institucionais ou temáticos e estimular que os resultados das suas pesquisas sejam publicados em periódicos de acesso aberto.

Ao estabelecer uma política, a instituição estará criando condições para o cumprimento de suas próprias estratégias (ABADAL et al, 2009). De acordo com Suber (2009), para que a política possa de fato garantir determinados resultados, ela deve contemplar algumas questões, tais como:

- se a política será uma recomendação ou um requisito;

- a estratégia que ela recomendará (se via verde ou via dourada);

\footnotetext{
4 "To maximize impact, minimize redundancy and speed scientific progress, authors and publishers should aim to make research easy to access".
} 
- o repositório onde deverá ser feito o depósito, em que tempo e o que deverá ser depositado;

- as permissões que deverão ser concedidas para as publicações fruto de uma pesquisa promovida ou financiada pela instituição;

- posicionamento sobre o embargo que porventura for estabelecido pelos editores científicos;

- sanções para o descumprimento da política.

Esses são alguns dos pontos apresentados e discutidos pelo autor como fundamentais para tais políticas, tanto para as universidades quanto para as agências de fomento. Suber (2009) declara que a política para a promoção do acesso aberto deve ser de caráter obrigatório (um requisito) para o financiamento de pesquisas. Ele justifica seu posicionamento com a experiência do National Institutes of Health (NIH), que iniciou em 2005 com uma política de recomendação e somente em 2007, quando se tornou obrigatória, é que as ações receberam grande quantidade de adesões.

Suber (2009) também recomenda que a estratégia em que a política deve focar é a via verde, ou seja, na criação e no fortalecimento dos repositórios institucionais. Quando a política institucional determina que os trabalhos devem ser publicados em revistas de acesso aberto, isso pode limitar o universo de escolha dos pesquisadores. Para defender sua posição, o autor argumenta que apenas $15 \%$ das revistas científicas são de acesso aberto. Entretanto, como discutido anteriormente, este dado pode ser questionado dependendo da região em que a pesquisa foi feita.

No estudo de Cerda Silva e Lara (2011), por exemplo, foi diagnosticado que 99\% das revistas científicas da América Latina possibilitam o acesso aberto. Estes dados são resultados de uma pesquisa das políticas editorias das revistas latino-americanas, feita por amostragem. Para a pesquisa foram selecionadas 292 revistas, que representam cerca de $17 \%$ do total das revistas disponíveis nos diretórios Scielo, Latindex e RedALyC. No entanto, apesar dos dados apontarem para um cenário favorável para o acesso aberto por meio das revistas científicas, há que se considerar que muitos são os autores da América Latina que publicam ou têm a intenção de publicar em revistas internacionais e de acesso restrito.

Suber (2009) aponta a necessidade da política em definir onde e quando será armazenado o trabalho fruto da pesquisa financiada pela instituição, mesmo que o editor que publicou o documento determine um tempo de embargo para a divulgação em outros meios. Este é um ponto importante da política, já que ao publicar um trabalho os autores 41

Enc. Bibli: R. Eletr. Bib. Ci. Inf., ISSN 1518-2924, Florianópolis, v. 17, n. esp. 2 - III SBCC, p.36-54, 2012. 
normalmente transferem para as revistas seus direitos patrimoniais sobre a obra, cabendo aos editores determinar se e quando poderão ser disponibilizados em acesso aberto os resultados de sua pesquisa. Quando uma política estipula um tempo máximo para o cumprimento de um embargo, ela fortalece as iniciativas que objetivam tornar acessível a informação científica e garante os interesses dos autores e da própria instituição.

Nesse contexto, segundo Abadal et al (2009), é importante que se faça conhecer amplamente as políticas que favoreçam o acesso aberto e em especial os diretórios especializados, pois isso possibilita o conhecimento de sua existência por parte da comunidade e cria condições para que as políticas já existentes sirvam de modelo para outras instituições. Para esses autores, “as principais fontes para conhecer a existência de políticas em favor do acesso aberto são Sherpa-Juliet e ROARMAP, assim como os valiosos apontamentos de Peter Suber" (p. 159).

Para ampliar a discussão sobre os diretórios especializados, a seguir serão analisados os três grandes serviços de divulgação de políticas para o acesso aberto: Sherpa/Juliet, Melibea e ROARMAP.

\section{SERVIÇOS DE DIFUSÃO DE POLÍTICAS PARA O ACESSO ABERTO}

Os diretórios especializados Sherpa/Juliet, Melibea e ROARMAP constituem-se em serviços para a difusão de políticas de instituições de pesquisa e de agências de fomento, colaborando, assim, com as iniciativas para o acesso aberto. $\mathrm{O}$ cumprimento dessa finalidade é possível porque além do registro das políticas, alguns dos serviços criam indicadores sobre o grau de favorecimento dessas políticas ao acesso aberto. As avaliações têm a intenção de contribuir com o desenvolvimento das políticas, ressaltando seus pontos fortes e deficiências, e servem para a elaboração de novas diretrizes.

\section{a. Sherpa/Juliet}

Sherpa/Juliet é o serviço desenvolvido pelo grupo SHERPA da University of Nottingham. Esse grupo tem desenvolvido várias ações de promoção e desenvolvimento do acesso aberto, entre as quais destacam-se o diretório Sherpa/Romeo para as políticas editoriais; o OpenDOAR, diretório de repositórios de acesso aberto; e o DRIVER, que trabalha para o desenvolvimento e aperfeiçoamento da infraestrutura para uma rede de repositórios. 
O serviço Sherpa/Juliet (Figura 2) se dedica à reunião e análise das políticas institucionais e foi criado, segundo o próprio diretório, porque as instituições de financiamento estavam cobrando cada vez mais a disponibilização em acesso aberto dos resultados de pesquisa. Assim sendo, esse serviço tem como função auxiliar na localização das políticas dos financiadores de pesquisas de diversas regiões, além de localizar e reunir as políticas destacando as principais condições de uma política ideal.

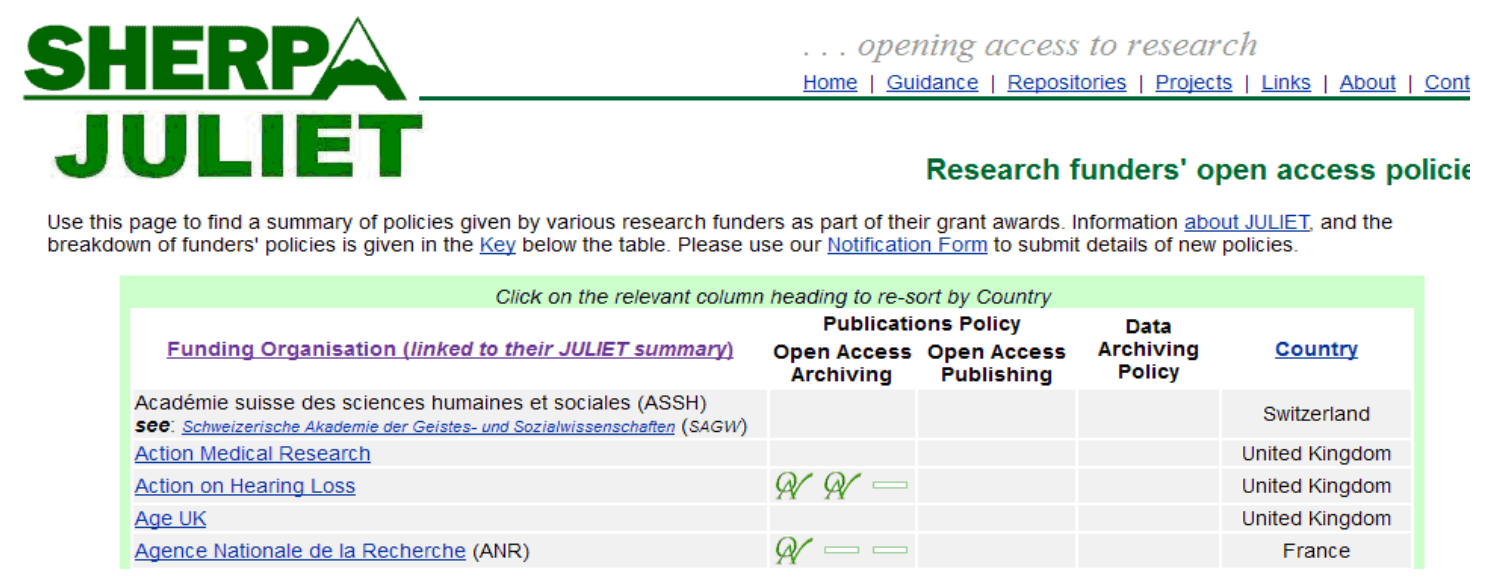

Figura 2: Sherpa/Juliet.

Fonte: http://www.sherpa.ac.uk/juliet/ (2012).

A metodologia para avaliar o quão perto ou distante as políticas estão do ideal é desenvolvida a partir da divisão da política em três partes principais. Essas partes correspondem às questões sobre arquivamento do documento, publicação do documento e arquivamento dos dados.

A primeira questão se refere ao armazenamento do documento onde foram publicados os resultados da pesquisa. Para ser bem avaliada nessa categoria, a política deve requisitar o depósito do documento sem restrição e acesso gratuito. $\mathrm{O}$ documento depositado deve ser a última versão do trabalho e deve estar disponível em texto completo. Sobre essa questão, ainda é avaliado se a política determina quando deve ser realizado o depósito. O modelo ideal recomendado pelo Sherpa/Juliet é aquele que dita que o depósito deve ser feito logo quando o trabalho for aceito para a publicação, ainda que esteja sob embargo determinado pelos editores.

A segunda questão é a que trata sobre como deve ser feita a publicação dos resultados da pesquisa. Segundo o serviço, a política é melhor qualificada se ela determina que o trabalho deve ser submetido a uma publicação de acesso aberto.

O serviço Sherpa/Juliet também avalia (terceira parte da análise) como a política trata a questão relacionada com o arquivamento dos dados. Nessa avaliação são determinados 43

Enc. Bibli: R. Eletr. Bib. Ci. Inf., ISSN 1518-2924, Florianópolis, v. 17, n. esp. 2 - III SBCC, p.36-54, 2012. 
como pontos ideais que os dados da pesquisa sejam arquivados em acesso aberto e que o depósito para o arquivamento seja realizado no prazo de cinco anos.

No diretório do serviço estão disponíveis informações estatísticas sobre os conselhos de pesquisa do Reino Unido e suas políticas sobre o acesso aberto, no entanto, tais dados limitam-se ao período de 2007 a 2008. Segundo informações do serviço, no período analisado, seis dos sete conselhos de pesquisa do Reino Unido financiaram pesquisas com uma política de acesso aberto. No entanto, faz-se necessário mencionar que a instituição que foi exceção à política de acesso aberto era responsável por cerca de $38 \%$ dos financiamentos. Essa análise demonstra que dos 1,2 bilhões de euros investidos em pesquisa na época e região, apenas 0,76 bilhões de euros foram investidos em pesquisas acompanhadas de uma política institucional que favorecia o acesso aberto (Figura 3).

\begin{tabular}{|c|c|c|c|}
\hline Funding Organisation (linked to their JULLET summary) & Grant Expenditure $(\{, 000)$ & Period & OA Policy \\
\hline Aits and Humanities Research Counci (AHRC) & 57,072 & $2007-2008$ & Yes \\
\hline Biotechnology and Biological Sciences Ressearch Council (BBSRC) & 202,699 & $2007-2008$ & Yes \\
\hline Economic and Social Research Counci (ESRC) & 115,494 & $2007-2008$ & Yes \\
\hline Engineering and Physical Sciences Research Counci (EPSRC) & 481,337 & $2007-2008$ & No \\
\hline Medical Research Counci (MRC) & 202,220 & $2007-2008$ & Yes \\
\hline Natural Environment Research Counci (NERC) & 81,685 & $2007-2008$ & Yes \\
\hline Science and Technology Facilities Council (STFC) & 102,895 & $2007-2008$ & Yes \\
\hline
\end{tabular}

3: Dados do Sherpa/Juliet.

Fonte: http://www.sherpa.ac.uk/juliet/financialstats.html (2012).

Figura

\section{b. Melibea}

O Melibea (Figura 4) é um serviço de difusão de políticas de acesso aberto, desenvolvido nos moldes do Sherpa/Juliet. O serviço é desenvolvido e mantido pelo grupo Acceso Abierto da Espanha, resultado de uma cooperação entre grupos da Universidade de Valencia, Universidade de Barcelona e da Universidade Aberta da Catalunha. Dentre as ações do Acceso Abierto, destaca-se o serviço Dulcinea, que reúne e divulga informações sobre a política editorial das revistas científicas da Espanha (serviço similar ao Sherpa/Romeo, mas restrito às revistas espanholas). 


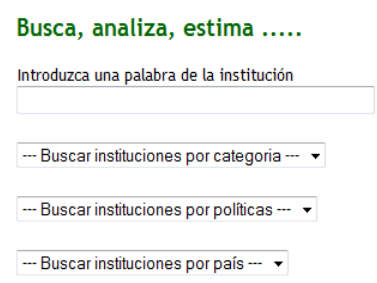

Segundo Meleros et al (2010), o objetivo do serviço Melibea é identificar e analisar as políticas existentes que favoreçam o acesso aberto, cuja pesquisa tenha sido financiada total ou parcialmente com recursos públicos. Para cumprir com esse objetivo, o serviço estabeleceu indicadores que permitem identificar quais são os pontos fortes e fracos de uma política, e elaborou uma metodologia para orientar as instituições a criarem suas diretrizes. O serviço oferece também uma ferramenta para que os seus conteúdos possam ser comparados.

O serviço se declara ser constituído de um diretório e um validador. O diretório representa o sistema que busca e analisa as políticas das instituições, já o validador é o responsável pela aplicação de um modelo que quantifica e pondera algumas questões da política. Esse validador é o responsável pela determinação da porcentagem de Acesso Aberto, uma espécie de nota indicada para a política.

As políticas existentes no diretório passam por duas divisões. A primeira divisão diz respeito à categoria da instituição, isto é, se ela é uma instituição acadêmica/de pesquisa ou se é uma entidade financiadora. A segunda divisão trata da política propriamente dita e é organizada em grupos diferentes: em políticas que funcionam como um requisito para o financiamento e as que funcionam como uma recomendação. Sobre essa questão, o serviço disponibiliza uma análise dos dados estatísticos que mostra que em 2010 quase $80 \%$ das políticas eram um requisito para o financiamento de pesquisa (Figura 5). 


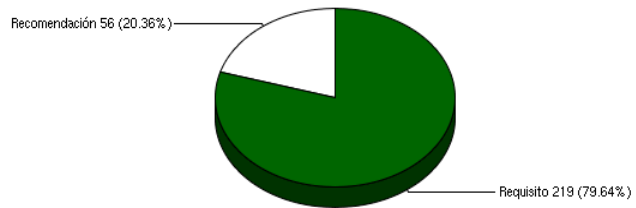

Figura 5: Dados do Melibea.

Fonte:

http://www.accesoabierto.net/politicas/default.php?directorio=politicas\&campo_1=POLITICA\&formato=grafic o\&titulo=Pol\%EDticas\%20OA:\%20Pol\%EDtica (2010).

\section{c. ROARMAP}

O ROARMAP (Figura 6) é o serviço de difusão de políticas institucionais de acesso aberto desenvolvido pelo grupo de trabalho eprints da Universidade de Southampton (ABADAL, 2009). O grupo é o desenvolvedor do sistema eprints, software livre para a criação e gestão de repositórios digitais.

\section{ROARMAP}

\begin{tabular}{|c|c|c|c|}
\hline COUNTRY & INSTITUTION(s) or FUNDER(s) & REPOSITORY URL(s) & Policy det \\
\hline $\begin{array}{l}\text { Slovenia } \\
\text { Thesis Mandate }\end{array}$ & University of Ljubljana, Faculty of Education & http://pefprints.pef.uni-li.si & $\underline{\text { Policy det: }}$ \\
\hline $\begin{array}{l}\text { Norway } \\
\text { Institutional Mandate }\end{array}$ & University of Oslo & $\begin{array}{l}\text { http://www.duo. uio.nol, http://www. cristin.no } \\
\text { las/WebObjects/cristin.woa }\end{array}$ & Policy deté \\
\hline$\frac{\text { United Kingdom }}{\text { Institutional Mandate }}$ & University of Reading & http://centaur.reading.ac.uk & $\underline{\text { Policy deté }}$ \\
\hline $\begin{array}{l}\text { Australia } \\
\text { Funder Mandate }\end{array}$ & National Health and Medical Research Council (NHMRC) & UNSPECIFIED & $\underline{\text { Policy deté }}$ \\
\hline $\begin{array}{l}\text { Australia } \\
\text { Institutional Mandate }\end{array}$ & Queensland University of Technology & http:/leprints.qut.edu.au/ & Policy deté \\
\hline $\begin{array}{l}\text { Australia } \\
\text { Sub-Institutional Mandate }\end{array}$ & University of Tasmania: School of Computing & http://eprints.comp.utas.edu.aul & Policy deté \\
\hline$\frac{\text { Australia }}{\text { Institutional Mandate }}$ & University of Tasmania & http://eprints.utas.edu.au/ & Policy deté \\
\hline $\begin{array}{l}\text { Australia } \\
\text { Funder Mandate }\end{array}$ & Australian Research Council & $\begin{array}{l}\text { http://leven.comp.utas.edu.au/AuseAccess } \\
\text { Ipmwiki.php?n=General.UniPolicies }\end{array}$ & $\underline{\text { Policy deté }}$ \\
\hline Australia & Mantinnal Hanlth and Madianl Dannareh Snumnil IMLMDOSI & hittp://leven comp.utas edu.au/AuseAccess & Donlin \\
\hline
\end{tabular}

Figura 6: ROARMAP.

Fonte: http://roarmap.eprints.org/ (2012).

O objetivo desse serviço é reunir as políticas institucionais que fazem referência ao depósito de publicações em repositórios de acesso aberto. As políticas reunidas pelo diretório 
são categorizadas $\mathrm{em}^{5}$ : institucional (política de uma determinada instituição), subinstitucional (política de um determinado setor da instituição), multi-institucional (política de várias instituições), e financiadoras (política de instituições financiadoras). As quatro categorias representam o tipo de instituição que elabora a política. O próprio serviço disponibiliza dados estatísticos de como os registros dessas políticas têm sido feitos ao longo do tempo e por tipo de instituição (Figura 7).

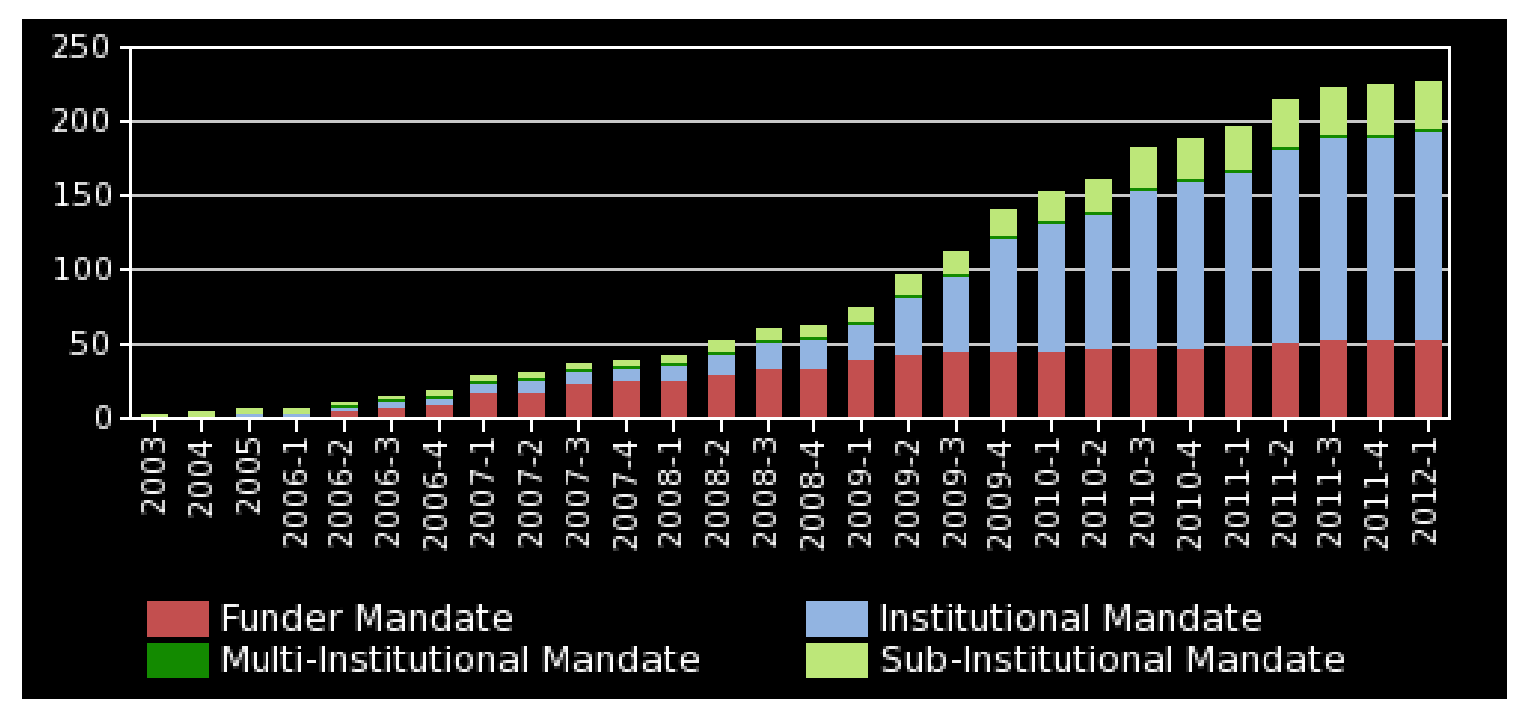

Figura 7: Dados do ROARMAP.

Fonte: http://roarmap.eprints.org/ (2012).

\section{ESTUDO COMPARATIVO}

Para realização do estudo comparativo entre os serviços apresentados neste trabalho, foram definidos cinco pontos para a comparação, quais sejam:

I Forma de apresentação das informações sobre as políticas, coletadas das instituições;

II Sistema de busca das políticas oferecido pelos serviços;

III Quantidade de políticas registradas no diretório de serviço;

IV Informações disponibilizadas pelo serviço sobre as políticas;

V Forma de avaliação das políticas pelo serviço.

Cada ponto será discutido, a seguir, conforme a numeração estabelecida anteriormente para cada tópico de avaliação.

\footnotetext{
${ }^{5}$ O serviço também considera políticas específicas para teses. Entretanto, para a discussão ora apresentada decidiu-se por não considerar a categoria teses por esta não ser da mesma natureza de informação das outras políticas.

47

Enc. Bibli: R. Eletr. Bib. Ci. Inf., ISSN 1518-2924, Florianópolis, v. 17, n. esp. 2 - III SBCC, p.36-54, 2012.
} 
I O primeiro ponto trata do modo como são apresentadas as informações coletadas por cada serviço em seus diretórios. Essa categoria é importante para que o usuário do serviço saiba como estão dispostas as informações e as políticas no sistema. Permite também, ao usuário, encontrar com mais facilidade a informação desejada.

a. O Sherpa/Juliet apresenta uma lista com todas as instituições cujas políticas estão registradas. A lista é apresentada na página inicial do site do serviço e os nomes das instituições são organizados alfabeticamente.

b. O Melibea não apresenta uma lista com todas as instituições na primeira página como o Sherpa/Juliet, mas oferece um sistema de busca que permite a localização das políticas.

c. O ROARMAP faz a junção das apresentações do Sherpa/Juliet e Melibea, disponibilizando uma lista das instituições, organizada de forma alfabética e por país, e ainda dispõe de um sistema de busca.

II Como apresentado na questão anterior, os serviços que apresentam mecanismos de busca das políticas no diretório são o Melibea e o ROARMAP. O Sherpa/Juliet não disponibiliza sistema de busca, no entanto, todas as políticas e suas informações estão dispostas na página inicial do serviço.

a. No Melibea é possível realizar a busca por nome, categoria (instituição de pesquisa ou entidade financiadora) ou país da instituição, além de uma busca pelo tipo de política (recomendação ou requisito).

b. No ROARMAP a busca pode ser realizada pelo país e nome da instituição ou pelo tipo de política (política institucional, política subinstitucional, política multi-institucional e política de instituição financiadora). O serviço ainda conta com o sistema de busca simples e avançada que permite relacionar dois ou mais campos.

III Os serviços Sherpa/Juliet e ROARMAP são mais antigos do que o Melibea, sendo este último construído a partir de uma análise dos outros dois serviços. No entanto, o serviço Melibea tem quantitativamente mais registros de políticas comparado ao Sherpa/Juliet e 
menos registros do que o ROARMAP. Atualmente, existem 312 políticas de aproximadamente 41 regiões registradas no ROARMAP, 275 políticas de cerca de 41 países no Melibea e 130 de políticas de 18 regiões no Sherpa/Juliet.

IV A quantidade de informações sobre as políticas que estes serviços oferecem foi analisada neste estudo comparativo. Esse ponto é relevante para serviços como esses, pois permite ao usuário conhecer alguns pontos essenciais da política sem a necessidade de acessála.

a. O Sherpa/Juliet informa o armazenamento e a publicação dos trabalhos em acesso aberto, além de oferecer informações de como cada política trata dos dados e resultados das pesquisas. As informações de cada política são relacionadas com o nome da instituição e seu país de origem.

b. O Melibea disponibiliza aproximadamente dezoito informações sobre a instituição e sua política, além de oferecer uma ferramenta para medição sobre a promoção do acesso aberto. Ainda, informa se a política é de caráter obrigatório ou recomendada, a sua data de homologação, informações sobre como deve ser o acesso, e também como, quando e onde deve ser feito o armazenamento dos trabalhos produzidos pela instituição.

c. O serviço que menos apresenta informações sobre as políticas registradas é o ROARMAP, que disponibiliza apenas o nome, o país e a URL da instituição. Para obter informações sobre uma política é necessário acessá-la e fazer a leitura do documento.

V O sistema de avaliação proposto pelos serviços é importante para esse contexto porque estabelece indicadores para a orientação de criação e edição das políticas. A avaliação também se mostra relevante para a comparação entre os conteúdos de forma a possibilitar um cenário das políticas em relação ao acesso aberto. Os serviços que oferecem sistema de avaliação de políticas são o Sherpa/Juliet e o Melibea, portanto o serviço ROARMAP não será analisado nessa categoria.

A avaliação do Sherpa/Juliet é menos complexa do que a do Melibea, no entanto, contempla um ponto importante para as políticas institucionais de informação que aponta para como devem ser tratados os dados da pesquisa. A partir da divisão em três grandes categorias de elementos importantes para uma política, foram determinadas as questões a serem 49

Enc. Bibli: R. Eletr. Bib. Ci. Inf., ISSN 1518-2924, Florianópolis, v. 17, n. esp. 2 - III SBCC, p.36-54, 2012. 
avaliadas em cada categoria. Dessa forma, o serviço atribui um símbolo para cada questão que a política contempla.

b. O Melibea apresenta um sistema de avaliação para o acesso aberto atribuindo pontuação a vinte e quatro questões do questionário de registro das políticas. As respostas são pontuadas dentro de um sistema de ponderação de nota, ou seja, dependendo do tipo de instituição ou política, uma questão pode receber mais ou menos pontos. Essa metodologia de avaliação gera um indicador de porcentagem apontando o quanto a política favorece o acesso aberto às publicações das pesquisas de uma instituição.

Todas as questões sobre a comparação dos serviços, discutidas neste tópico, podem ser visualizadas no quadro a seguir (Quadro 1).

\begin{tabular}{|c|c|c|c|}
\hline Serviço & $\begin{array}{c}\text { a. } \\
\text { SHERPA/JULIET } \\
\text { Sherpa }\end{array}$ & $\begin{array}{c}\text { b. } \\
\text { MELIBEA } \\
\text { Acesso Abierto }\end{array}$ & $\begin{array}{c}\text { c. } \\
\text { ROARMAP } \\
\text { Eprints }\end{array}$ \\
\hline $\begin{array}{c}\text { I Forma de } \\
\text { apresentação das } \\
\text { informações }\end{array}$ & $\begin{array}{l}\text { Apresenta uma lista das } \\
\text { instituições na página } \\
\text { inicial do site. }\end{array}$ & $\begin{array}{l}\text { Apresenta um sistema de } \\
\text { busca com opções de } \\
\text { refinamento. }\end{array}$ & $\begin{array}{l}\text { Apresenta uma lista na } \\
\text { página inicial do site e um } \\
\text { sistema de busca com } \\
\text { opções de refinamento. }\end{array}$ \\
\hline $\begin{array}{l}\text { II Sistema de } \\
\text { busca }\end{array}$ & $\begin{array}{l}\text { Não possui sistema de } \\
\text { busca. }\end{array}$ & $\begin{array}{l}\text { Busca por: nome da } \\
\text { instituição, categoria da } \\
\text { instituição, país da } \\
\text { instituição e tipo de } \\
\text { política. }\end{array}$ & $\begin{array}{l}\text { Busca por: nome da } \\
\text { instituição, país da } \\
\text { instituição e tipo de } \\
\text { instituição. }\end{array}$ \\
\hline $\begin{array}{c}\text { III Quantidade de } \\
\text { políticas }\end{array}$ & Aproximadamente 130. & Aproximadamente 275 . & Aproximadamente 312 . \\
\hline
\end{tabular}




\begin{tabular}{|c|c|c|c|}
\hline $\begin{array}{l}\text { IV Informações } \\
\text { disponibilizadas } \\
\text { pelo serviço sobre } \\
\text { as políticas }\end{array}$ & $\begin{array}{l}\text { Sobre a instituição: } \\
\text { - Nome; } \\
\text { - País. }\end{array}$ & $\begin{array}{l}\text { Sobre a instituição: } \\
\text { - Nome; } \\
\text { - Categoria; } \\
\text { - URL. }\end{array}$ & $\begin{array}{l}\text { Sobre a instituição: } \\
\text { - Nome; } \\
\text { - País; } \\
\text { - URL. }\end{array}$ \\
\hline & $\begin{array}{l}\text { Sobre a política: } \\
\text { - Avaliação do serviço. }\end{array}$ & $\begin{array}{l}\text { Sobre a política: } \\
\text { - Avaliação do serviço; } \\
\text { - Tipo de política; } \\
\text { - Data. }\end{array}$ & \\
\hline $\begin{array}{c}\text { V Forma de } \\
\text { avaliação das } \\
\text { políticas }\end{array}$ & $\begin{array}{l}\text { Atribuição de um } \\
\text { símbolo para cada } \\
\text { quesito satisfeito pela } \\
\text { política. }\end{array}$ & $\begin{array}{l}\text { Oferece uma ferramenta } \\
\text { que pontua a política e faz } \\
\text { uma estimativa do quanto } \\
\text { a política favorece o } \\
\text { acesso aberto. }\end{array}$ & $\begin{array}{l}\text { Não possui sistema de } \\
\text { avaliação das políticas. }\end{array}$ \\
\hline
\end{tabular}

\section{CONSIDERAÇÕES FINAIS}

Com o objetivo de tornar conhecidas as iniciativas que as instituições de fomento promovem à pesquisa por meio de suas políticas, foram desenvolvidos serviços para difusão das políticas para o acesso aberto, que reúnem, organizam, analisam e difundem as políticas das instituições de pesquisa e das agências de fomento. As políticas institucionais deverão contemplar, entre outros pontos, uma orientação aos autores para que publiquem os resultados de suas pesquisas em periódicos de acesso aberto e/ou para que façam o depósito de suas publicações em repositórios institucionais ou temáticos. Com esses serviços, além de ser ampliada a visibilidade das políticas e as ações das instituições, também são criados indicadores que dizem respeito ao que uma instituição pode estabelecer para contribuir para o acesso aberto à informação científica. 
Um estudo sobre os serviços de difusão das informações sobre o acesso aberto pode contribuir com a ampliação do conhecimento sobre os serviços nas discussões e iniciativas da área, além de possibilitar que se tenha uma noção clara do que se pode esperar de cada um dos serviços estudados. Neste trabalho foram estudados os serviços Sherpa/Juliet, Melibea e ROARMAP. Pôde-se verificar que os serviços apresentam similaridades em alguns elementos e diferenças em outros, mas de modo geral não fogem à sua finalidade de divulgar as políticas existentes e contribuir para a criação de novas políticas para a promoção do acesso aberto.

Nos três serviços analisados foram identificadas apenas três políticas brasileiras para a promoção do acesso aberto. Esse cenário aponta para a necessidade da criação de iniciativas similares à desses serviços. Um serviço brasileiro de difusão de políticas ainda poderia contribuir para a criação de políticas nacionais para o acesso aberto que sejam coerentes com a realidade nacional, além de dar visibilidade àquelas já criadas. 


\section{REFERÊNCIAS}

ABADAL, E. et al. Políticas institucionales para el fomento del acceso abierto: tipología y buenas prácticas. Bollettino AIB, v. 49, n. 2, p. 159-170, jun. 2009. Disponível em: < http://eprints.rclis.org/handle/10760/13565>. Acesso em: abr. 2012.

BAPTISTA, A.; COSTA, S.; KURAMOTO, H.; RODRIGUES,E. Comunicação científica: o papel da Open Archives Initiative no contexto do Acesso Livre. Encontros Bibli: Revista Eletrônica de Biblioteconomia e Ciência da Informação, Florianópolis, n. Especial, $1^{\mathrm{o}}$ sem. 2007. Disponível em: <www.periodicos.ufsc.br/index.php/eb>. Acesso em: mar 2012.

CERDA SILVA, Alberto; LARA, Juan Carlos. Políticas editoriales de publicaciones académicas en línea en Latinoamérica. Revistas Abiertas, 2011. Disponível em: http://www.revistasabiertas.com/wp-content/uploads/Informe-

Politicas_Editoriales_de_Publicaciones.pdf ONG Derechos Digitales. Acesso em: 3 fev. 2012.

DEARING, Matthew T. The rise of open access scientific publishing. Disponível em: $<\mathrm{http}$ //www.science20.com/dynamic_patterns_research_journal/rise_open_access_scientific_ publishing-86745>. Acesso em: 14 fev. 2012.

LAAKSO, Mikael et al. O desenvolvimento da Publishing Open Access Journal: 19932009. PLoS ONE 6 (6). Disponível em: $\mathrm{http}: / /$ translate.googleusercontent.com/translate_c?hl=pt-

BR\&langpair $=$ en $\% 7 \mathrm{Cpt} \&$ rurl=translate.google.com.br\&twu=1\&u=http://www.plosone.org/ar ticle/info\%253Adoi\%252F10.1371\%252Fjournal.pone.0020961\&usg=ALkJrhihckQ5bMH9i CljwyyloSbKmvV4NQ. Acesso em: 08 mar. 2012.

LEITE, F. Como gerenciar e ampliar a visibilidade da informação científica brasileira: repositórios institucionais de acesso aberto. Brasília: IBICT, 2009. 120 p.

LAWRENCE, Steve. Free online availability substantially increases a paper's impact. Nature webdebates. Disponível em:

$<$ http://www.nature.com/nature/debates/e-access/Articles/lawrence.html $>$. Acesso em: mar. 2012 .

LIMA, Marcia H. T. de Figueredo. Consequências do movimento pelo livre acesso - Open Access - e o direito à informação científica. In: SAYÃO, Luis et al (Org.). Implantação e gestão de repositórios institucionais: políticas, memória, livre acesso e preservação. Salvador: EDUFBA, 2009. p. 219-230.

MELERO, R. et al. The open access validator: an approach to create and weight your open access policy. [2010?]. 1 poster, color. Disponível em: $<$ http://www.accesoabierto.net/sites/accesoabierto.net/files/poster-OR2010.pdf $>$. Acesso em: 5 out. 2011.

MUELLER, Suzana Pinheiro Machado. A comunicação científica e o movimento de acesso livre ao conhecimento. Revista Ciência da Informação, Brasília, v. 35, n. 2, p. 27-38, maio/ago. 2006. 
SHERPA JULIET. Research funders' open access policy. Disponível em: $<$ http://www.sherpa.ac.uk/juliet/>. Acesso em: mar. 2012.

SUBER, Peter. Open access policy options for funding agencies and universities. Welcome to the SPARC Open Access Newsletter, n. 130. fev. 2009. Disponível em: $<$ http://www.earlham.edu/ peters/fos/newsletter/02-02-09.htm>. Acesso em: 14 fev. 2012.

TOMAÉL, M. I.; SILVA, T. E. Repositórios institucionais: diretrizes para políticas de informação. In: ENCONTRO NACIONAL DE PESQUISA EM CIÊNCIA DA INFORMAÇÃO, 8., 28 a 31 de outubro de 2007. Anais... Salvador, 2007. Disponível em: $<$ http://www.enancib.ppgci.ufba.br/artigos/GT5--142.pdf>. Acesso em: 20 nov. 2011. 